\title{
Fast subsonic combustion as a free-interface problem
}

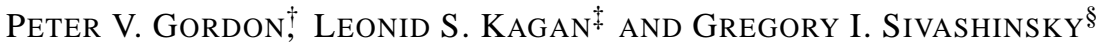 \\ School of Mathematical Sciences, Sackler Faculty of Exact Sciences, \\ Tel-Aviv University, Tel-Aviv 69978, Israel
}

[Received 11 March 2002 and in revised form 27 October 2002]

\begin{abstract}
The paper is concerned with the recently identified fast, yet subsonic, combustion waves occurring in obstacle-laden (e.g. porous) systems and driven not by thermal diffusivity but rather by the draginduced diffusion of pressure. In the framework of a quasi-one-dimensional formulation where the impact of obstacles is accounted for through a frictional drag term, an asymptotic expression for the wave propagation velocity $D$ is derived. The propagation velocity is controlled by the temperature $\left(T_{+}\right)$at the entrance to the reaction zone rather than at its exit $\left(T_{b}\right)$ as occurs in deflagrative combustion. The evaluated $D\left(T_{+}\right)$dependence allows description of the subsonic detonation in terms of a free-interface problem. The latter is found to be dynamically akin to the problem of gasless combustion known for its rich pattern-forming dynamics.
\end{abstract}

\section{Introduction}

Premixed gas combustion is the combustion of gaseous reactants which are perfectly mixed prior to ignition. The most distinctive feature of premixed combustion is its ability to assume the form of a self-sustained reaction wave propagating subsonically or supersonically at a well-defined speed. Apart from their technological relevance, combustion waves constitute a truly fascinating dynamical system, displaying an amazingly rich variety of phenomena such as non-uniqueness of possible propagation regimes, their birth (ignition) and destruction (extinction), chaotic self-motion and fractal-like growth, various hysteretic transitions, etc.

One of the most effective practices in the theoretical exploration of combustion waves is their description in terms of a free-interface problem where the reaction zone is considered as infinitely thin compared to the other length-scales involved.

The current study is concerned with the formulation and analysis of a free-interface problem associated with the recently identified new mode of subsonic combustion arising in hydraulically resisted flows (e.g. porous beds), and where the combustion wave is driven by the drag-induced diffusion of pressure, rather than thermal diffusivity as occurs in conventional unconfined flames. In the simplest case (the so-called small-heat release approximation) the emerging free-interface problem is described by a single filtration equation (Sec. 6),

$$
\Pi_{\tau}=\gamma \nabla^{2} \Pi
$$

where $\Pi$ is the reduced pressure that vanishes far ahead of the reactive interface $f=0$, and approaches unity far behind; $\gamma$ is the ratio of specific heats. At the interface the following matching

†Email: peterg@math.uchicago.edu

Email: kagan@cfg.eng.tau.ac.il

${ }^{\S}$ Corresponding author. Email: grishas@ post.tauac.il 
conditions are held:

$$
\gamma[\nabla \Pi \cdot \mathbf{n}]=-\Phi(\Pi)=-v_{f}, \quad[\Pi]=0,
$$

where $\Phi(\Pi)$ is the Arrhenius-type source intensity, $\mathbf{n}=\nabla f /|\nabla f|$ is the normal to the interface, and $v_{f}=-f_{\tau} /|\nabla f|$ its normal velocity. For all its seeming simplicity the system (1.1)-(1.2) is quite rich dynamically exhibiting both regular and complex behavior (Sec. 2).

The paper is organized as follows. Section 2 yields a brief physical overview of combustion in hydraulically resisted flows, singling out the calculation of the equilibrium planar wave as a crucial step in the passage to the free-interface formulation. Sections 3, 4 deal with the planar wave and evaluation of the associated propagation velocity. The latter involves a non-conventional eigenvalue problem solved by an asymptotic separation of variables in an appropriate phase-plane. In Section 5 the relations obtained for the planar wave are extended over quasi-steady and quasiplanar configurations. As a result one ends up with a reduced model for pressure-driven combustion based on a free-interface formulation. To test its dynamical well-posedness the model is simulated numerically (Sec. 6).

\section{Physical overview}

As is well known there are basically two mechanisms controlling propagation of combustion waves in gaseous mixtures: molecular transport and adiabatic compression. The first mechanism pertains to deflagrative combustion and the second to detonation. Normally the adiabatic compression is provided by the shock and the resulting combustion wave (detonation) spreads at a supersonic speed. In conventional detonation the shock and adiabatic compression are two inseparable ingredients. This coupling, however, is not inevitable. In hydrodynamically resisted flows, as those developing in porous beds, the burning velocity may fall significantly below its thermodynamic, ChapmanJouguet (CJ) value, and under certain conditions the propagation may well become subsonic and therefore shockless $[12,13,16,20]$. The shockless propagation still involves pressure peaks and is sustained by adiabatic compression which is now spread by the drag-induced diffusion of pressure. The transition from supersonic to subsonic propagation normally occurs in a gradual manner, e.g. by dilution of the mixture. In the near-sonic propagation the presence or absence of shocks is not crucial and there is no major distinction between supersonic and subsonic combustion. The subsonic mode emerges as a continuous extension of the supersonic one and hence may well be referred to as subsonic detonation, two words that one would have thought could never be connected! The idea of extending the concept of the detonation over the subsonic domain is not new and has already been discussed by Mitrofanov in his 1987 review on non-classical combustion waves [16]. In the concluding lines of the paper the author wrote, "It would be appropriate to extend the notion of detonation over a certain subsonic range of wave velocities $\left(D<a_{0}\right)$ where there is a continuous in $D$ passage to this domain with the preservation of pressure and density peaks within the front". It is interesting that the issue was raised by the experimentalists ten years ahead of the theoretical substantiation of the phenomenon. In [3, 4] employing the classical Zeldovich-Fanno [22] formulation, it was shown that the conventional and subsonic modes are actually two special cases of the general drag-affected detonation and in the velocity-drag plane belong to the same curve of dynamically feasible states (Fig. 1). The conventional CJ-detonation corresponds to the limit of low hydraulic resistance while the subsonic detonation to the limit of strong resistance.

In the subsonic detonation, due to the strong temperature dependence of the reaction rate, the bulk of the reaction is localized in a narrow sublayer of the overall wave structure controlled by 
the pressure diffusivity and the wave speed. This observation suggests the possibility of describing the pertinent dynamical system in terms of a free-interface problem, advantageous for theoretical explorations. To make such a formulation possible one needs a relation between the intensity of the localized reaction rate and the interface temperature. This, in turn, requires an analytical expression for the reaction wave speed relative to the background flow-field. It transpires, however, that the rational evaluation of this parameter cannot be carried out along the lines of the classical ZeldovichFrank-Kamenetsky approach employed in the deflagration theory [21]. The point is that in subsonic detonation the temperature within the reaction zone undergoes an abrupt increase rather than being a slowly varying function as occurs in deflagrative combustion. As a result the traditional multiplescale technique developed for analytical tackling of deflagrations appears unsuitable here. In the current paper the difficulty is resolved by the asymptotic separation of variables in the pressureconcentration phase-plane.

Curiously enough, in a certain parameter range, the emerging free-interface problem becomes functionally identical to the problem of gasless combustion (self-sustained high-temperature synthesis) known for its rich pattern forming dynamics, involving, among other things, galloping and spinning structures as well as period-doubling cascades and chaos $[1,2,6,10,14]$.

\section{Model}

To describe a reaction wave spreading through a hydraulically resisted flow the presence of obstacles will be accounted for by means of the velocity-dependent drag-force term added to the momentum equation. The current study is focused on the subsonic detonation only where $D<a_{0}$ (Fig. 1). Moreover, we shall consider the limit $D \ll a_{0}$, pertinent to strong hydraulic resistance. In this case the impact of inertial effects may be discarded and the system of governing equations may be written as

$$
\begin{aligned}
& \left(c_{v} \rho T\right)_{t}+\nabla\left(c_{v} \rho T \mathbf{u}+p \mathbf{u}\right)=Q W, \quad \text { energy, } \\
& (\rho C)_{t}+\nabla(\rho C \mathbf{u})=-W, \quad \text { concentration, } \\
& \rho_{t}+\nabla(\rho \mathbf{u})=0, \quad \text { continuity, } \\
& \nabla p=\mathbf{f}, \quad \text { momentum, } \\
& p=\left(c_{p}-c_{v}\right) \rho T, \quad \text { state. }
\end{aligned}
$$

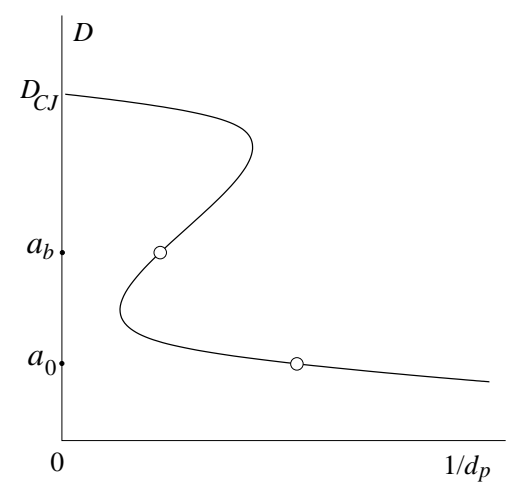

FIG. 1. Detonation velocity $(D)$ vs. reciprocal of the particle diameter $\left(d_{p}\right)[3,4] . D_{C J}, a_{0}, a_{b}$ correspond to the ChapmanJouguet detonation and sound speeds in the fresh and burnt gas, respectively. 
Here $W$ is the reaction rate defined by a one-step first-order Arrhenius kinetics,

$$
W=A \rho C \exp (-E / R T)
$$

where $E$ is a constant, specific to the reaction and called its activation energy, $R$ is the universal gas constant, $Q$ is the heat release, $C$ is the concentration of the deficient reactant, $A$ is the preexponential factor. Other notations are conventional.

The drag-force $\mathbf{f}$ is specified by the classical Darcy-Forchheimer equation [7, 8, 17],

$$
\mathbf{f}=-\left(\frac{\phi v}{K}\right) \rho \mathbf{u}-\left(\frac{\phi^{3} F}{\sqrt{K}}\right) \rho \mathbf{u}|\mathbf{u}| .
$$

Here $v$ is the kinematic viscosity of the fluid, $\phi$ is the porosity, i.e. the fraction of the bulk volume of porous bed occupied by void space. $K$ is the permeability (conductivity) of the porous bed with respect to permeation by a fluid. This parameter is independent of the nature of the fluid but depends on the geometry of the medium. $F$ is the Forchheimer coefficient controlling the impact of inertial effects ignored in Darcy's equation pertinent to the creeping flow limit. For packed-sphere beds, $K$ and $F$ are expressed in terms of the porosity $\phi$ and the particle diameter $d_{p}$ by (see [8])

$$
K=\frac{\phi^{3} d_{p}^{2}}{\Gamma(1-\phi)^{2}}, \quad F=\frac{\Sigma}{\Gamma^{1 / 2} \phi^{3 / 2}},
$$

where $\Gamma, \Sigma$ are empirical constants. According to [8], $\Gamma=150$ and $\Sigma=1.75$.

To reduce the number of parameters involved, the effects due to molecular transport and heat losses are discarded. Aside from the obvious advantages for physical analysis (conservation of enthalpy), the adiabatic limit may serve as quite a legitimate approximation away from the quenching point where the Reynolds analogy is violated and the momentum loss emerges as a dominating influence.

\section{Traveling-wave solution}

As mentioned in Section 2 the key point in converting the above formulation into a free-interface problem is the evaluation of the propagation velocity $D$ of the associated traveling-wave solution.

In the reference frame attached to the steady planar wave, Eqs. (3.1)- 3.4 ) yield

$$
\begin{aligned}
& \mathrm{d}\left[\rho(u-D) c_{v} T+p u\right] / \mathrm{d} x=Q W, \\
& \mathrm{~d}[\rho(u-D) C] / \mathrm{d} x=-W, \\
& \mathrm{~d}[\rho(u-D)] / \mathrm{d} x=0, \\
& \mathrm{~d} p / \mathrm{d} x=f .
\end{aligned}
$$

The wave is assumed to propagate through an initially quiescent homogeneous premixture whose temperature, pressure, density, and deficient reactant concentration are regarded as prescribed. Hence the boundary conditions are

$$
\begin{aligned}
& T(\infty)=T_{0}, \quad C(\infty)=C_{0}, \quad \rho(\infty)=\rho_{0} \\
& p(\infty)=p_{0}=\left(c_{p}-c_{v}\right) \rho_{0} T_{0}, \quad u(\infty)=0 .
\end{aligned}
$$


Far behind the wave, due to the flow deceleration and the reactant consumption,

$$
u(-\infty)=0, \quad C(-\infty)=0,
$$

and the global integration of the enthalpy equation, $(\mathrm{Eq} 4.1)+Q(\mathrm{Eq} 4.2)$,

$$
\mathrm{d}\left[\rho(u-D)\left(c_{v} T+Q C\right)+p u\right] / \mathrm{d} x=0,
$$

yields

$$
T(-\infty)=T_{0}+Q C_{0} / c_{v}=T_{b}, \quad p(-\infty)=\left(c_{p}-c_{v}\right) \rho_{0} T_{b}=p_{b} .
$$

Thus, due to the boundary condition (4.8) and the absence of heat losses, the final temperature and pressure of burnt gas appear to be identical to those reached in the constant volume adiabatic explosion.

Integration of 4.3 and 4.7 subject to the boundary conditions 4.5, , 4.6, 4.8 allows the flow velocity, temperature and the specific volume, $v=1 / \rho$, to be expressed in terms of the pressure and concentration. Specifically,

$$
\begin{aligned}
u & =D\left[1-\left(T p_{0} / T_{0} p\right)\right], \\
v & =c_{p}\left(1-\gamma^{-1}\right)(T / p), \quad \gamma=c_{p} / c_{v}, \\
T & =\gamma^{-1} T_{b}+\left(1-\gamma^{-1}\right) T_{0}\left(p / p_{0}\right)-\gamma^{-1}\left(T_{b}-T_{0}\right)\left(C / C_{0}\right) .
\end{aligned}
$$

At high activation energies $\left(E / R T_{b} \gg 1\right)$ the reaction zone shrinks, and on the length scale larger than its width the reaction rate may be treated as a localized source,

$$
W \simeq M \delta(x)
$$

where $M$ is the source intensity (to be found). Given 4.12, Eqs. (4.1)-(4.4) imply the following jump conditions at the interface:

$(x=0)$

$$
\begin{aligned}
& {\left[\rho(u-D) c_{v} T+p u\right]=M Q,} \\
& {[\rho(u-D) C]=-M,} \\
& {[\rho(u-D)]=0,} \\
& {[p]=0 .}
\end{aligned}
$$

Since

$$
C(x>0)=C_{0} \quad \text { and } \quad C(x<0)=0,
$$

and by (4.3)- (4.5) and $\rho(u-D)=-\rho_{0} D$, Eq. (4.14) readily implies

$$
M=C_{0} \rho_{0} D,
$$

where at this stage $D$ remains undetermined.

Outside the reaction zone the overall problem is easily tractable and one ends up with the following set of basic profiles. At $x<0$ (burnt gas),

$$
C=0, \quad T=T_{b}, \quad v=v_{b}=v_{0}, \quad u=0, \quad p=p_{b} .
$$


At $x \geqslant 0$ (fresh mixture),

$$
\begin{aligned}
C & =C_{0}, \quad T=\gamma^{-1} p_{0}^{-1} T_{0}\left(p_{0}+(\gamma-1) p(x)\right), \\
v & =\gamma^{-2}(\gamma-1) c_{p} p_{0}^{-1} T_{0}\left(p_{0}+(\gamma-1) p(x)\right) / p(x), \\
u & =\gamma^{-1} D\left(p(x)-p_{0}\right) / p(x),
\end{aligned}
$$

where $p(x)$ is governed by the equation

$$
\mathrm{d} p / \mathrm{d} x=f(v(p), u(p)), \quad p(0)=p_{0} .
$$

At the upstream (unburnt) side of the reaction zone,

$$
T=T_{+}=T_{0}+\left(1-\gamma^{-1}\right)\left(T_{b}-T_{0}\right) .
$$
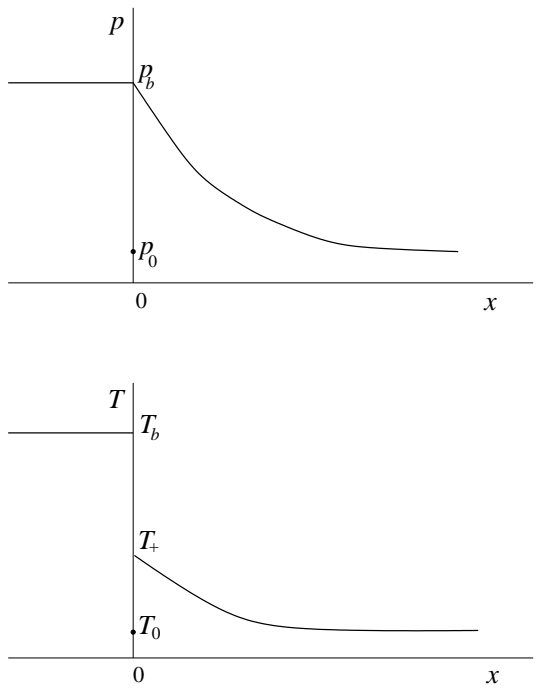

FIG. 2. Profiles of pressure ( $p$ ) and temperature $(T)$ in subsonic detonation (outer view).

Thus within the reaction zone the temperature undergoes a sharp increase from $T_{+}$to $T_{b}$ (Fig. 2). As has been shown by Goldfarb et al. [11], $T_{+}$plays a pivotal role in controlling the reaction time scale $\tau$,

$$
\tau \sim \exp \left(E / R T_{+}\right)
$$

not dissimilar to the situation with the post-shock (Neumann) temperature in supersonic detonation [22].

\section{Evaluation of the propagation velocity}

To evaluate the propagation velocity $D$, it is helpful to introduce a set of scaled quantities,

$$
\begin{aligned}
& \Theta=\left(T-T_{0}\right) /\left(T_{b}-T_{0}\right), \quad \Pi=\left(p-p_{0}\right) /\left(p_{b}-p_{0}\right), \quad \Psi=1-C / C_{0}, \quad V=v / v_{0}, \\
& U=u / D, \quad \xi=x / l, \quad \tau=D t / l, \quad \sigma=T_{0} / T_{b}, \quad q=(1-\sigma) / \sigma .
\end{aligned}
$$


Here the length scale $l$ is defined by

$$
l=q a_{0}^{2} K^{1 / 2} / \gamma \phi^{3} F D^{2},
$$

where $a_{0}=\sqrt{\gamma\left(c_{p}-c_{v}\right) T_{0}}$ is the velocity of sound in the unburnt gas. The scaled version of Eqs. (4.2) and 4.4 thus becomes

$$
\begin{aligned}
\Lambda^{3} \frac{\mathrm{d} \Psi}{\mathrm{d} \xi} & =-\frac{1}{V} \Omega(\Psi, \Theta), \\
\frac{\mathrm{d} \Pi}{\mathrm{d} \xi} & =-\frac{U(\kappa+|U|)}{V},
\end{aligned}
$$

where according to 4.9 - 4.11 ,

$$
\begin{aligned}
& \Theta=\left(1-\gamma^{-1}\right) \Pi+\gamma^{-1} \Psi, \quad V=(1+q \Theta) /(1+q \Pi), \quad U=q(\Pi-\Psi) / \gamma(1+q \Pi), \\
& \Omega=(1-\Psi) \exp \left[\beta\left(\Theta-\left(1-\gamma^{-1}\right)\right) /(\sigma+(1-\sigma) \Theta)\right],
\end{aligned}
$$

is the scaled reaction rate, where $\beta=(1-\sigma) E / R T_{+}$is the effective Zeldovich number. $\Lambda$ is the scaled propagation velocity defined by

$$
\Lambda^{3}=\gamma \phi^{3} F D^{3} \exp \left(E / R T_{+}\right) / A q a_{0}^{2} K^{1 / 2},
$$

and

$$
\kappa^{-1}=\phi^{2} F D K^{1 / 2} / v
$$

is the effective Reynolds number. Eqs. 5.3 - 5.4 are considered jointly with the boundary conditions

$$
\Psi(\infty)=0, \quad \Psi(-\infty)=1, \quad \Pi(\infty)=0, \quad \Pi(-\infty)=1
$$

To circumvent the familiar cold-boundary difficulty we shall introduce an auxiliary ignition temperature $\Theta=\Theta^{*}$ and redefine $\Omega$ so that

$$
\Omega=0 \quad \text { at } 0<\Theta<\Theta^{*} .
$$

For high enough $\beta$ the final result should not depend on the specifics of the truncation.

At $\Theta<\Theta^{*}$, due to the absence of diffusion, $\Psi=0$, and hence by Eq. 5.5 , $\Theta=\left(1-\gamma^{-1}\right) \Pi$. At the ignition point, therefore,

$$
\Pi=\Pi^{*}=\Theta^{*} /\left(1-\gamma^{-1}\right) .
$$

Assuming that $U>0$ and hence $\mathrm{d} \Pi / \mathrm{d} \eta<0$, the boundary conditions (5.9) immediately imply that $\Pi<1$ and by 5.11), $\Theta^{*}<1-\gamma^{-1}$. Hence, $\Pi$ may now be employed as a new independent variable reducing Eqs. (5.3)-(5.4) to a single first-order ODE,

$$
\Lambda^{3} \frac{\mathrm{d} \Psi}{\mathrm{d} \Pi}=-\frac{\Omega(\Psi, \Theta(\Psi, \Pi))}{U^{2}(\Psi, \Pi)+\kappa(\Lambda) U(\Psi, \Pi)},
$$

considered jointly with the boundary conditions

$$
\Psi\left(\Pi^{*}\right)=0, \quad \Psi(1)=1 .
$$


Being overdetermined, the problem 5.12 - 5.13 should produce both $\Psi(\Pi)$ and the scaled propagation velocity $\Lambda$.

For further analysis it is helpful to recast Eq. (5.12) as

$$
\Lambda^{3} H(\Psi, \Pi) \mathrm{d} \Psi=G(\Psi, \Pi) \mathrm{d} \Pi
$$

where

$$
\begin{aligned}
G(\Psi, \Pi)= & \gamma q^{-2}(1+q \Pi)^{2} \exp \left(\frac{\beta\left(1-\gamma^{-1}\right)(\Pi-1)}{\sigma+(1-\sigma)\left(\left(1-\gamma^{-1}\right) \Pi+\gamma^{-1} \Psi\right)}\right), \\
H(\Psi, \Pi)= & \frac{(\Pi-\Psi)\left(\Pi-\Psi+\gamma q^{-1} \kappa(\Lambda)(1+q \Pi)\right)}{\gamma(1-\Psi)} \\
& \times \exp \left(-\frac{\beta \gamma^{-1} \Psi}{\sigma+(1-\sigma)\left(\left(1-\gamma^{-1}\right) \Pi+\gamma^{-1} \Psi\right)}\right) .
\end{aligned}
$$

Considering $G$ as a function of $\Pi$, and $H$ as a function of $\Psi, \Lambda^{3}$ is determined by the relation

$$
\Lambda^{3} J=I,
$$

where

$$
I=\int_{\Pi^{*}}^{1} G(\Psi(\Pi), \Pi) \mathrm{d} \Pi \text { and } \quad J=\int_{0}^{1} H(\Psi, \Pi(\Psi)) \mathrm{d} \Psi .
$$

At large Zeldovich numbers, $\Psi$ is close to zero everywhere except for a small vicinity of $\Pi=1$ where it sharply raises to unity. The asymptotic evaluation of the integrals 5.17 then yields (see Appendix)

$$
\begin{aligned}
& I=\frac{\gamma(1+q)^{2}\left(\sigma+(1-\sigma)\left(1-\gamma^{-1}\right)\right)}{\beta q^{2}\left(1-\gamma^{-1}\right)}+O\left(\frac{\ln \beta}{\beta^{3 / 2}}\right) \\
& J=\frac{\left(1+\gamma q^{-1}(1+q) \kappa(\Lambda)\right)\left(\sigma+(1-\sigma)\left(1-\gamma^{-1}\right)\right)}{\beta}+O\left(\frac{\ln \beta}{\beta^{3 / 2}}\right) .
\end{aligned}
$$

Substituting (5.18)-(5.19) into 5.16 one readily obtains

$$
\Lambda^{3}=\frac{\gamma(1+q)^{2}}{q^{2}\left(1-\gamma^{-1}\right)\left(1+\gamma q^{-1}(1+q) \kappa(\Lambda)\right)}+O\left(\frac{\ln \beta}{\sqrt{\beta}}\right) .
$$

To test the rate of convergence, Figure 3 shows the numerical solution of the eigenvalue problem 5.12, 5.13) corresponding to $\kappa=0, \Pi^{*}=0.25, \sigma=0.15(q=5.667), \gamma=1.2,1.3,1.4$, and $5<\beta<50$. For example, at $\gamma=1.3, T_{0}=295^{\circ} \mathrm{K}$ and $E / R T_{b}=15$, as follows from Eq. (4.22, $T_{+}=681^{\circ} \mathrm{K}$ and hence $\beta=(1-\sigma) E / R T_{+}=37$. In this case $\Lambda(37)=1.95, \Lambda(\infty)=1.98$ and thus $(\Lambda(\infty)-\Lambda(37)) / \Lambda(\infty)=0.015$, which is quite a small error.

Finally, upon returning to the original dimensional parameters, 5.20 yields

$$
\left(\frac{F \phi^{3} \sigma(1-\sigma)(\gamma-1)}{\gamma A a_{0}^{2} \sqrt{K}}\right) D^{3}+\left(\frac{\phi \nu \sigma(\gamma-1)}{A a_{0}^{2} K}\right) D^{2}=\exp \left(-\frac{E}{R T_{+}}\right) .
$$




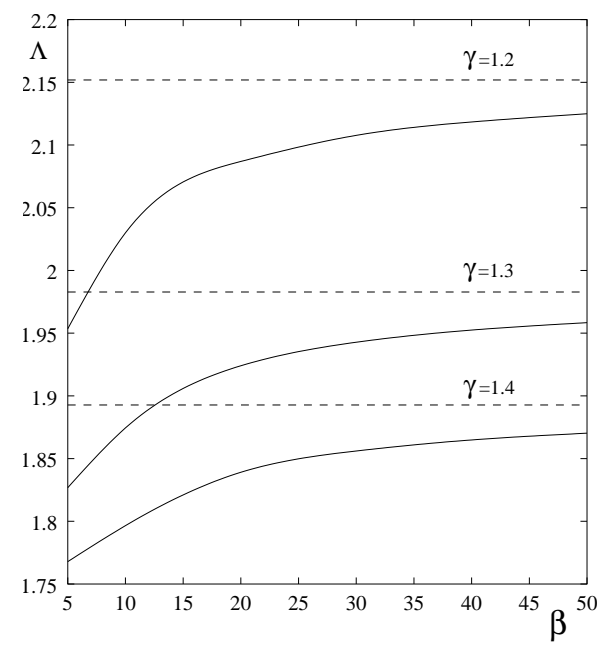

FIG. 3. Scaled propagation velocity $(\Lambda)$ vs. scaled activation energy $(\beta)$ evaluated for $\gamma=1.2,1.3,1.4, \kappa=0$ and $\sigma=0.15$. Broken lines correspond to the asymptotics 5.20 .

At $A=10^{8} \mathrm{~s}^{-1}, E / R=10,000^{\circ} \mathrm{K}, T_{0}=295^{\circ} \mathrm{K}, a_{0}=350 \mathrm{~m} / \mathrm{s}, d_{p}=0.25 \mathrm{~cm}, v=0.15 \mathrm{~cm}^{2} / \mathrm{s}$, $\phi=0.4, \sigma=T_{0} / T_{b}=0.15, \gamma=1.3$, Eqs. 4.22, 5.21 yield $T_{+}=681^{\circ} \mathrm{K}, D=74 \mathrm{~m} / \mathrm{s}$, $l=23 \mathrm{~cm}$ and $\kappa=0.026$. For fast reaction waves spreading through porous beds the parameter $\kappa$ (5.8) is normally quite small, which would justify omission of the linear (Darcy) term in Eq. (3.7) and, hence, the quadratic term in Eq. 5.21). As a result one ends up with a cubic-root dependence on the Arrhenius exponent,

$$
D \sim \exp \left(-E / 3 R T_{+}\right)
$$

Note that in the subsonic detonation the propagation speed is controlled by the temperature $\left(T_{+}\right)$at the entrance to the reaction zone rather than at its exit $\left(T_{b}\right)$ as occurs in deflagrative combustion.

\section{Free-interface problem and its numerical validation}

The consumption rate, $C_{0} \rho_{0} D\left(T_{+}\right)$4.18, evaluated for steady planar waves will apparently hold also for the waves whose reaction zone is merely quasi-steady and quasi-planar. In this situation $T_{+}$is no longer obliged to coincide with its thermodynamic value (4.22), but should be determined by the overall dynamics of the system. Due to the high activation energy even a mild variation in $T_{+}$may strongly affect $D\left(T_{+}\right)$. This approach, long and successfully employed in deflagrative combustion, allows description of subsonic detonation in terms of a free-interface problem. The corresponding set of governing equations may thus be written as

$$
\begin{aligned}
& \left(c_{v} \rho T\right)_{t}+\nabla \cdot\left(c_{v} \rho T \mathbf{u}+p \mathbf{u}\right)=Q C_{0} \rho_{0} D\left(T_{+}\right) \delta_{F}, \\
& (\rho C)_{t}+\nabla \cdot(\rho C \mathbf{u})=-C_{0} \rho_{0} D\left(T_{+}\right) \delta_{F} .
\end{aligned}
$$

Here $\delta_{F}$ is the surface $\delta$-function with the subscript $F$ labeling the flame-interface, $F(\mathbf{x}, t)=0$. Eqs. (6.1), (6.2) should be considered jointly with Eqs. (3.3)- 3.5), (4.17), (5.21). 


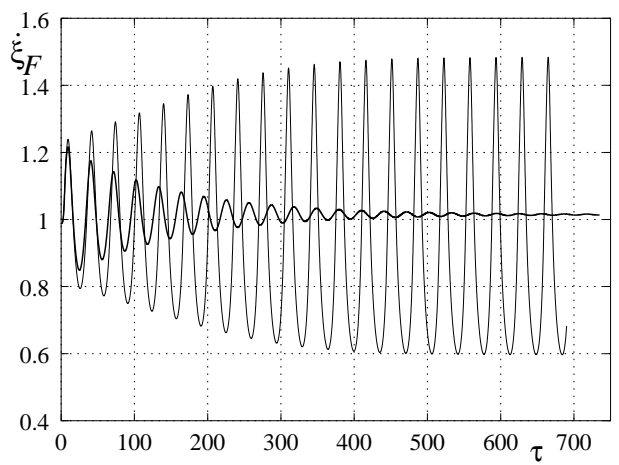

FIG. 4. Scaled propagation velocity $\left(\dot{\xi}_{F}\right)$ versus scaled time $(\tau)$ calculated for $\gamma=1.3, \sigma=0.15, \kappa=0.025, \beta=19$ and 20 . Bold and thin curves correspond to the intrinsically stable $(\beta=19)$ and galloping $(\beta=20)$ propagation.
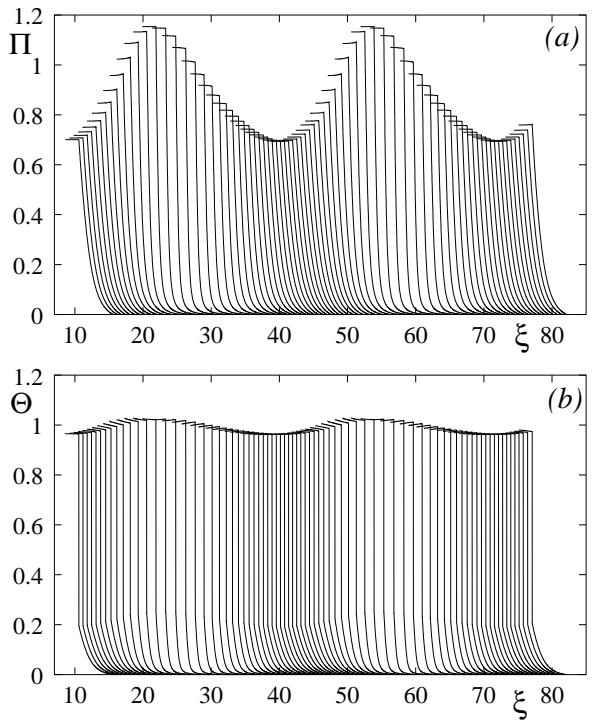

FIG. 5. Galloping mode of subsonic detonation. Profiles of scaled pressure $\Pi(a)$ and temperature $\Theta(b)$ at several consecutive instants of time; $\gamma=1.3, \sigma=0.15, \kappa=0.025, \beta=20$.

Due to (4.17), integration of Eq. 6.2) across the interface yields

$$
\rho_{+}\left(V_{F}-\mathbf{u}_{+} \cdot \mathbf{n}\right)=\rho_{0} D\left(T_{+}\right),
$$

where $V_{F}=-F_{t} /|\nabla F|$ is the normal velocity of the interface, and $\mathbf{n}=\nabla F /|\nabla F|$ is its unit normal. One thus ends up with a model involving only three equations 3.3, 3.4) and 6.1).

Although the constructed free-interface formulation involves discontinuities, the drag-induced pressure diffusivity exerts a regularizing influence which should make the system well-posed dynamically, i.e. free of pathological instabilities as occurs, for example in the Darrieus-Landau [15] or Zaidel's [9] problems also dealing with combustion interfaces. To test this point the current model was simulated numerically for the simplest case of one-dimensional propagation (Figs. 4, 5). 
As one might anticipate, at sufficiently low Zeldovich numbers $(\beta)$ the equilibrium traveling-wave solution appears to be stable. Yet there is a critical value $\beta=\beta_{c r}(\sigma, \gamma)$ above which the traveling wave loses its stability and the propagation occurs in an oscillatory (galloping) mode. Otherwise the model is quite well-behaved dynamically as far as pathological instabilities are concerned.

A more systematic approach to the stability question requires consideration of an eigenvalue problem for a system of linear ODEs with variable coefficients. The latter stem from the nonlinear convective terms and constitute a major obstacle for an analytical tackling of the problem. This difficulty, however, vanishes if one considers the limit of small-heat-release $(\sigma \rightarrow 1)$ which, while suppressing convective transport, preserves much of the problem's character including its discontinuities. In this limit, as has recently been shown [2], the system of governing equations (3.1)-3.7) reduces to a piecewise-linear problem for the pressure. In the appropriately chosen units (5.1) the resulting model reads

$$
\begin{aligned}
\Pi_{\tau} & =\gamma \nabla^{2} \Pi+\Phi(\Pi) \delta_{f}, \\
v_{f} & =\Phi(\Pi) .
\end{aligned}
$$

Here $v_{f}=-f_{\tau} /|\nabla f|$ is the normal velocity of the interface, $f(\xi, \tau)=0$, and

$$
\Phi(\Pi)=\exp \left(\frac{\hat{\beta}(\Pi-1)}{2(\hat{\sigma}+(1-\hat{\sigma}) \Pi)}\right)
$$

with

$$
\hat{\beta}=\frac{(1-\sigma)\left(1-\gamma^{-1}\right)\left(E / R T_{b}\right)}{1-\gamma^{-1}(1-\sigma)}, \quad \hat{\sigma}=\frac{\gamma \sigma}{\gamma-1+\sigma} \quad(\hat{\sigma}<1) .
$$

Eq. 6.4 should be considered jointly with the condition $\Pi \rightarrow 0$ far ahead of the advancing interface.

The reduced model, as one may notice, is formally identical to the free-interface model for gasless combustion (self-propagating high-temperature synthesis) if $\Pi$ is regarded as the scaled temperature; $\hat{\beta}$, Zeldovich number and $\hat{\sigma}$, the unburnt/burnt phase temperature ratio $(\hat{\sigma}<1)$. In this context the model has been explored energetically in recent years and there is a sizeable volume of analytical and numerical results on its intrinsic dynamics. The latter, among other things, involves galloping and spinning waves as well as period-doubling cascades and chaos [1, 2, 6, 10, 14].

Note that for the reduced model $(6.4)-(6.7)$ the basic traveling-wave solution becomes oscillatingly unstable as soon as the scaled Zeldovich number $\hat{\beta} \sim\left(1-\gamma^{-1}\right)(1-\sigma)\left(E / R T_{b}\right)$ exceeds some critical value [2]. Thus, for all the distinction in the underlying physics, the instability criteria for both subsonic and supersonic detonation $[5,18]$ appear to be quite similar in form.

\section{Concluding remarks}

The multiplicity of drag-affected detonation regimes shown in Figure 1 is qualitatively similar to that found by Stewart and Yao [19] in curved detonations. The major difference is that in the latter case the additional (curvature) source term appears in the conservation of mass equation instead of the momentum equation and the emerging detonation regimes appear to be essentially supersonic.

In general, drag-force based models can represent fast reaction waves in hydraulically resisted flows only in some average sense. In the subsonic low Mach number detonation discussed in this paper the developing reaction wave width,

$$
l_{w}=\left(p_{b}-p_{0}\right)(\mathrm{d} p(+0) / \mathrm{d} x)^{-1},
$$


is normally much larger than the porous bed particle diameter $d_{p}$ and may easily extend over tens of centimeters [13]. For example, for the parameter set considered in Section $4, l_{w}=74 d_{p}=18.5 \mathrm{~cm}$. In such a situation the reaction wave is expected to be only mildly distorted by the porous bed granules, and the adopted formulation is likely to be valid not only qualitatively but also quantitatively.

\section{Acknowledgments}

These studies were supported in part by the U.S.-Israel Binational Science Foundation under Grant No. 98-00374, the Israel Science Foundation under Grants Nos. 67-01 and 574-00 the German-Israel Foundation under Grant No. 695-15.10.01, and the European Community Program RTN-HPRN-CT2002-00274.

\section{Appendix}

In this section we construct an a priori estimate for the solution $\Psi(\Pi)$ of the problem (5.12), 5.13) and evaluate $\Lambda$ at $\beta \rightarrow \infty$.

Step 1. Estimate of $\Psi(\Pi)$. Suppose that $\Lambda \sim O(1)$. Then there exist constants $\hat{M} \sim O(1)$ and $\check{M} \sim O(1)$ such that $\hat{M}>\Lambda>\check{M}$. Therefore $\hat{\kappa}>\kappa(\Lambda)>\check{\kappa}$, where $\hat{\kappa}=\kappa(\check{M})$ and $\check{\kappa}=\kappa(\hat{M})$. Then an upper solution of Eq. 5.12) with the second boundary condition 5.13 is defined as a solution of the equation

$$
\frac{\mathrm{d} \tilde{\Psi}}{\mathrm{d} \Pi}=\tilde{M} S(\tilde{\Psi}, \Pi)
$$

where $S(\Psi, \Pi)$ is any function satisfying the condition

$$
S(\Psi, \Pi) \leqslant G(\Psi, \Pi) / H(\Psi, \Pi) \quad \text { for any } \Pi \in\left(\Pi^{*}, 1\right) \text { and } \Psi \geqslant 0
$$

and $\tilde{M}$ is any positive number such that $\tilde{M} \leqslant \hat{M}^{-3}$.

The solution $\tilde{\Psi}$ will obviously be as close to the true solution $\Psi$ as $G / H$ is close to $S$. Thus to have some idea about $\Psi$ via $\tilde{\Psi}$ one has to ensure that $S$ retains the basic qualitative features of $G / H$. Such a specific choice may be made if we observe that for any $\Pi \in\left(\Pi^{*}, 1\right)$ and $\Psi \geqslant 0$,

$$
\begin{gathered}
\frac{(1-\Psi)(1+q \Pi)^{2}}{(\Pi-\Psi)\left(\Pi-\Psi+\gamma q^{-1} \kappa(\Lambda)(1+q \Pi)\right)} \geqslant \frac{1}{1+\gamma q^{-1} \hat{\kappa}(1+q)}, \\
\exp \left(\frac{\beta\left(1-\gamma^{-1}\right)(\Pi-1)}{\sigma+(1-\sigma)\left(\left(1-\gamma^{-1}\right) \Pi+\gamma^{-1} \Psi\right)}\right) \geqslant \exp \left(\frac{\beta\left(1-\gamma^{-1}\right)(\Pi-1)}{\sigma+(1-\sigma)\left(1-\gamma^{-1}\right) \Pi^{*}}\right), \\
\exp \left(\frac{\beta \gamma^{-1} \Psi}{\sigma+(1-\sigma)\left(\left(1-\gamma^{-1}\right) \Pi+\gamma^{-1} \Psi\right)}\right) \geqslant \exp \left(\beta \gamma^{-1} \Psi\right),
\end{gathered}
$$

and set

$$
S=\frac{\gamma^{2}}{q^{2}\left(1+\gamma q^{-1}(1+q) \hat{\kappa}\right)} \exp \left(\frac{\beta\left(1-\gamma^{-1}\right)(\Pi-1)}{\sigma+(1-\sigma)\left(1-\gamma^{-1}\right) \Pi^{*}}\right) \exp \left(\beta \gamma^{-1} \Psi\right) .
$$

As for $\tilde{M}$ we only assume that $\tilde{M} \sim O(1)$. 
Separating variables in A.1 with $S$ defined by A.6 and taking into account the boundary condition (5.13) at $\Pi=1$, we have

$$
\begin{aligned}
& \int_{1}^{\tilde{\Psi}} \exp \left(-\beta \gamma^{-1} s\right) \mathrm{d} s \\
& \quad=\frac{\gamma^{2}}{q^{2}\left(1+\gamma q^{-1}(1+q) \hat{\kappa}\right)} \tilde{M} \int_{1}^{\Pi} \exp \left(\frac{\beta\left(1-\gamma^{-1}\right)(s-1)}{\sigma+(1-\sigma)\left(1-\gamma^{-1}\right) \Pi^{*}}\right) \mathrm{d} s,
\end{aligned}
$$

which gives

$$
\tilde{\Psi}(\Pi)=-\frac{1}{\gamma^{-1} \beta} \ln \left(\tilde{M}_{1}\left(1-\exp \left(\frac{\beta\left(1-\gamma^{-1}\right)(\Pi-1)}{\sigma+(1-\sigma)\left(1-\gamma^{-1}\right) \Pi^{*}}\right)\right)+\exp \left(-\gamma^{-1} \beta\right)\right),
$$

where $\tilde{M}_{1}=\tilde{M}\left(\sigma+(1-\sigma)\left(1-\gamma^{-1}\right) \Pi^{*}\right) / q^{2}\left(1+\gamma q^{-1}(1+q) \hat{\kappa}\right) \gamma^{-1}\left(1-\gamma^{-1}\right)$. If we also require that

$$
\tilde{M}<q^{2}\left(1+\gamma q^{-1}(1+q) \hat{\kappa}\right) \gamma^{-1}\left(1-\gamma^{-1}\right) /\left(\sigma+(1-\sigma)\left(1-\gamma^{-1}\right) \Pi^{*}\right)
$$

(which is always possible) then $\tilde{\Psi}(\Pi)$ will be positive for all $\Pi \in\left(\Pi^{*}, 1\right)$ and thus $\mathrm{A} .8$ is an upper solution of (5.14) considered with both conditions (5.13).

The solution (A.8) possesses an interesting property (easily checked by direct substitution) that $\Psi \sim O\left(\beta^{-1}\right)$ at $\Pi=1-\beta^{-1}$. Moreover $\Psi \sim O\left(\beta^{-1} \ln \beta\right)$ at $\Pi=1-\beta^{-n}$ where $n>0$ is any fixed number. These observations imply that

$$
\begin{array}{ll}
\Psi(\Pi) \leqslant a_{1} \beta^{-1} & \text { for } \Pi \leqslant 1-a_{2} \beta^{-1}, \\
\Psi(\Pi) \leqslant a_{3} \beta^{-1} \ln \beta & \text { for } \Pi \leqslant 1-a_{4} \beta^{-n}, \text { for any } n>0 .
\end{array}
$$

Henceforth all constants $a_{i}$ are assumed to be of order of unity.

It is also important to note that jointly with $\Psi(\Pi)$ we can also consider the inverse function $\Pi(\Psi)$ due to its monotonicity. The lower solution for this problem is merely an inversion of $\tilde{\Psi}(\Pi)$, which implies

$$
\begin{array}{ll}
1-\Pi \leqslant a_{5} \beta^{-1} & \text { for } \Psi \geqslant a_{6} \beta^{-1} \\
1-\Pi \leqslant a_{7} \beta^{-1} \ln \beta & \text { for } \Psi \geqslant a_{8} \beta^{-n}, \text { for any } n>0
\end{array}
$$

Step 2. Evaluation of $\Lambda$. To calculate $\Lambda$ we have to obtain approximate values of the integrals $I$ and $J$ defined by (5.17).

Consider $I$ first. Let us rewrite this integral as a sum of three terms,

$$
\begin{aligned}
I= & I_{1}+I_{0}+I_{-1} \\
= & \int_{\Pi^{*}}^{1-1 / \sqrt{\beta}} \gamma q^{-2}(1+q \Pi)^{2} \exp \left(\frac{\beta\left(1-\gamma^{-1}\right)(\Pi-1)}{\sigma+(1-\sigma)\left(\left(1-\gamma^{-1}\right) \Pi+\gamma^{-1} \Psi(\Pi)\right)}\right) \mathrm{d} \Pi \\
& +\int_{1-1 / \sqrt{\beta}}^{1-1 / \beta^{2}} \ldots+\int_{1-1 / \beta^{2}}^{1} \cdots
\end{aligned}
$$

The first and last terms in this expression are readily estimated as follows:

$$
\left|I_{-1}\right| \leqslant a_{9} \beta^{-2}, \quad\left|I_{1}\right| \leqslant a_{10} \exp \left(-a_{11} \sqrt{\beta}\right),
$$


while $I_{0} \sim \beta^{-1}$. The main contribution to $I$ therefore comes from $I_{0}$. Let us calculate this integral. Note that by A.10, $\Psi \leqslant a_{3} \beta^{-1} \ln \beta$ for $\Pi \in\left(1-\beta^{-1 / 2}, 1-\beta^{-2}\right)$ and therefore

$$
\begin{aligned}
\exp \left(\frac{\beta\left(1-\gamma^{-1}\right)(\Pi-1)}{\sigma+(1-\sigma)\left(\left(1-\gamma^{-1}\right) \Pi+\gamma^{-1} \Psi(\Pi)\right)}\right) \\
=\left(1+O\left(\frac{\ln \beta}{\sqrt{\beta}}\right)\right) \exp \left(\frac{\beta\left(1-\gamma^{-1}\right)(\Pi-1)}{\sigma+(1-\sigma)\left(1-\gamma^{-1}\right) \Pi}\right)
\end{aligned}
$$

which allows us to write

$$
I_{0}=\tilde{I}_{0}\left(1+O\left(\frac{\ln \beta}{\sqrt{\beta}}\right)\right)
$$

where

$$
\tilde{I}_{0}=\gamma q^{-2} \int_{1-1 / \sqrt{\beta}}^{1-1 / \beta^{2}}(1+q \Pi)^{2} \exp \left(\frac{\beta\left(1-\gamma^{-1}\right)(\Pi-1)}{\sigma+(1-\sigma)\left(1-\gamma^{-1}\right) \Pi}\right) \mathrm{d} \Pi .
$$

The last integral can be calculated using the Laplace technique which gives

$$
\tilde{I}_{0}=\frac{\gamma(1+q)^{2}\left(\sigma+(1-\sigma)\left(1-\gamma^{-1}\right)\right)}{\beta q^{2}\left(1-\gamma^{-1}\right)}+O\left(\frac{1}{\beta^{2}}\right) .
$$

Thus,

$$
I=\frac{\gamma(1+q)^{2}\left(\sigma+(1-\sigma)\left(1-\gamma^{-1}\right)\right)}{\beta q^{2}\left(1-\gamma^{-1}\right)}+O\left(\frac{\ln \beta}{\beta^{3 / 2}}\right) .
$$

The second integral $J$ can be calculated using the same procedure. First we rewrite it as a sum

$$
\begin{aligned}
J= & J_{-1}+J_{0}+J_{1} \\
= & \int_{0}^{1 / \beta^{2}} \gamma^{-1} \frac{(\Pi(\Psi)-\Psi)\left(\Pi-\Psi+\gamma q^{-1} \kappa(\Lambda)(1+q \Pi)\right)}{1-\Psi} \\
& \times \exp \left(\frac{-\beta \gamma^{-1} \Psi}{\sigma+(1-\sigma)\left(\left(1-\gamma^{-1}\right) \Pi(\Psi)+\gamma^{-1} \Psi\right)}\right) \mathrm{d} \Psi+\int_{1 / \beta^{2}}^{1 / \sqrt{\beta}} \ldots+\int_{1 / \sqrt{\beta}}^{1} \ldots
\end{aligned}
$$

The terms $J_{-1}$ and $J_{1}$ are estimated as

$$
\left|J_{-1}\right| \leqslant a_{12} \beta^{-2}, \quad\left|J_{1}\right| \leqslant a_{13} \exp \left(-a_{14} \sqrt{\beta}\right),
$$

and $J_{0} \sim \beta^{-1}$ is the dominant term in $J$. Using A.12 we conclude that for $\Psi \in\left(\beta^{-2}, \beta^{-1 / 2}\right)$,

$$
\frac{(\Pi(\Psi)-\Psi)\left(\Pi-\Psi+\gamma q^{-1} \kappa(\Lambda)(1+q \Pi)\right)}{1-\Psi}=1+\gamma q^{-1}(1+q) \kappa(\Lambda)+O\left(\frac{\ln \beta}{\beta}\right),
$$

and

$$
\begin{aligned}
\exp \left(\frac{-\beta \gamma^{-1} \Psi}{\sigma+(1-\sigma)\left(\left(1-\gamma^{-1}\right) \Pi(\Psi)+\gamma^{-1} \Psi\right)}\right) \\
=\left(1+O\left(\frac{\ln \beta}{\sqrt{\beta}}\right)\right) \exp \left(\frac{-\beta \gamma^{-1} \Psi}{\sigma+(1-\sigma)\left(\left(1-\gamma^{-1}\right)+\gamma^{-1} \Psi\right)}\right) .
\end{aligned}
$$


Thus $J_{0}$ can be represented as

$$
J_{0}=\tilde{J}_{0}\left(1+O\left(\frac{\ln \beta}{\sqrt{\beta}}\right)\right)
$$

where

$\tilde{J}_{0}=\gamma^{-1}\left(1+\gamma q^{-1}(1+q) \kappa(\Lambda)\right) \int_{1 / \beta^{2}}^{1 / \sqrt{\beta}} \exp \left(\frac{-\beta \gamma^{-1} \Psi}{\sigma+(1-\sigma)\left(\left(1-\gamma^{-1}\right)+\gamma^{-1} \Psi\right)}\right) \mathrm{d} \Psi$.

Employing the Laplace technique we obtain

$$
\tilde{J}_{0}=\frac{\left(1+\gamma q^{-1}(1+q) \kappa(\Lambda)\right)\left(\sigma+(1-\sigma)\left(1-\gamma^{-1}\right)\right)}{\beta}+O\left(\frac{1}{\beta^{2}}\right),
$$

and hence

$$
J=\frac{\left(1+\gamma q^{-1}(1+q) \kappa(\Lambda)\right)\left(\sigma+(1-\sigma)\left(1-\gamma^{-1}\right)\right)}{\beta}+O\left(\frac{\ln \beta}{\beta^{3 / 2}}\right) .
$$

Substituting A.19, A.27) into 5.16) we finally obtain

$$
\Lambda^{3}=\frac{\gamma(1+q)^{2}}{q^{2}\left(1-\gamma^{-1}\right)\left(1+\gamma q^{-1}(1+q) \kappa(\Lambda)\right)}+O\left(\frac{\ln \beta}{\sqrt{\beta}}\right) .
$$

Since $\Lambda \sim O(1)$, our a priori assumption concerning its scaling is fully corroborated and therefore A.28 yields a true asymptotics as $\beta \rightarrow \infty$.

\section{REFERENCES}

1. Bayliss, A., \& Matkowsky, B. J. Two routes to chaos in solid fuel combustion. SIAM J. Appl. Math. 50 (1990), 437-459. MR 91b:80020 Zbl 0696.76082

2. Brailovsky, I., FrAnkel, M., \& SiVAShinsKy, G. I. Galloping and spinning modes of subsonic detonation. Combust. Theory Modelling 4 (2000), 47-60. MR 1752 120 Zbl 0966.76098

3. Brailovsky, I., \& Sivashinsky, G. Hydraulic resistance and multiplicity of detonation regimes. Combust. Flame 122 (2000), 130-138.

4. BRAILOVSKY, I., \& SivAShinsKy, G. I. Effects of momentum and heat losses on the multiplicity of detonation regimes. Combust. Flame 128 (2002), 191-196.

5. Clavin, P., \& He, L. Acoustic effects in the nonlinear oscillations of planar detonations. Phys. Rev. E 53 (1996), 4778-4784.

6. Dimitrou, P., Puszynski, J., \& HlaVačEK, V. On the dynamics of equations describing gasless combustion in condensed systems. Combust. Sci. Tech. 68 (1989), 101-111.

7. Dullien, F. A. L. Porous Media: Fluid Transport and Pore Structure. 2nd ed., Academic Press, San Diego (1992).

8. Ergun, S. Fluid flow through packed columns. Chem. Engrg. Progr. 48 (1952), 89-94.

9. ERPEnbeck, J. J. Structure and stability of the square-wave detonation. Proc. Combust. Inst. 9 (1963), $442-453$

10. Frankel, M., Roytburd, V., \& Sivashinsky, G. Complex dynamics generated by a sharp interface model of self-propagation high-temperature synthesis. Combust. Theory Modelling 2 (1998), 479-496. Zbl 0934.80003 
11. Goldfarb, I., Kuzmenko, G., \& Goldshtein, V. Structure of a pressure driven flame in porous media, Phys. Lett. A 251 (1999), 394-403.

12. Lyamin, G. A., Mitrofanov, V. V., Pinaev, A. V., \& Subbotin, V. A. Propagation of gas explosion in channels with uneven walls and in porous media. Dynamics of Detonation in Gases and Dispersed Media, A. A. Borisov (ed.), Kluwer, Dordrecht (1991), 51-75.

13. Lyamin, G. A., \& Pinaev, A. V. Fast subsonic combustion of gases in an inert porous medium with smooth rise in the pressure in the wave. Combust. Expl. Shock Waves 23 (1987), 399-402.

14. Margolis, S. B. The transition to nonsteady deflagration in gasless combustion. Progr. Energy Combust. Sci. 17 (1991), 135-162.

15. Markstein, G. H. Nonsteady Flame Propagation, Pergamon, Oxford (1964).

16. Mitrofanov, V. V. Non-classical waves of detonation and deflagration. Mechanics and ScientificTechnological Progress, L. I. Sedov and G. G. Chernyi (eds.), Vol. 2, Nauka, Moscow (1987), 226-242 (in Russian). MR 89e:80011 Zbl 0825.76901

17. Nield, D. A., \& BeJAn, A. Convection in Porous Media. Springer, New York (1992). MR 2000b:76107 Zbl 0924.76001

18. ShCHELKIN, K. I. Instability of combustion and detonation of gases. Soviet Physics Uspekhi 8 (1960), 780-797.

19. Stewart, D. S., \& YAO, J. The normal detonation shock velocity-curvature relationship for materials with nonideal equation of state and multiple turning points. Combust. Flame 113 (1998), 224-235.

20. Vasiliev, A. A., Mitrofanov, V. V., \& Topchiyan, M. E. Detonation waves in gases. Combust. Expl. Shock Waves 23 (1987), 605-623.

21. Zeldovich, Y. B., Barenblatt, G. I., Librovich, V. B., \& Makhviladze, G. M. The Mathematical Theory of Combustion and Explosion. Plenum, New York (1985). MR 86b:80014

22. Zeldovich, Y. B., \& Kompaneets, A. S. Theory of Detonation. Academic Press, New York (1960). 